\title{
Which Dengue Vaccine Approach Is the Most Promising, and Should We Be Concerned about Enhanced Disease after Vaccination?
}

\section{The Path to a Dengue Vaccine: Learning from Human Natural Dengue Infection Studies and Vaccine Trials}

\author{
Aravinda M. de Silva ${ }^{1}$ and Eva Harris ${ }^{2}$ \\ ${ }^{1}$ Department of Microbiology and Immunology, University of North Carolina School of Medicine, Chapel Hill, \\ North Carolina 27599 \\ ${ }^{2}$ Division of Infectious Diseases and Vaccinology, School of Public Health, University of California, Berkeley, \\ Berkeley, California 94720-3370 \\ Correspondence: eharris@berkeley.edu
}

Dengue virus (DENV) is the most common arthropod-borne viral disease of humans. Although effective vaccines exist against other flaviviral diseases like yellow fever and Japanese encephalitis, dengue vaccine development is complicated by the presence of four virus serotypes and the possibility of partial immunity enhancing dengue disease severity. Several live attenuated dengue vaccines are being tested in human clinical trials. Initial results are mixed, with variable efficacy depending on DENV serotype and previous DENV exposure. Here, we highlight recent discoveries about the human antibody response to DENV and propose guidelines for advancing development of safe and effective dengue vaccines.

\section{GREAT DEBATES}

What are the most interesting topics likely to come up over dinner or drinks with your colleagues? Or, more importantly, what are the topics that don't come up because they are a little too controversial? In Immune Memory and Vaccines: Great Debates, Editors Rafi Ahmed and Shane Crotty have put together a collection of articles on such questions, written by thought leaders in these fields, with the freedom to talk about the issues as they see fit. This short, innovative format aims to bring a fresh perspective by encouraging authors to be opinionated, focus on what is most interesting and current, and avoid restating introductory material covered in many other reviews.

The Editors posed 13 interesting questions critical for our understanding of vaccines and immune memory to a broad group of experts in the field. In each case, several different perspectives are provided. Note that while each author knew that there were additional scientists addressing the same question, they did not know who these authors were, which ensured the independence of the opinions and perspectives expressed in each article. Our hope is that readers enjoy these articles and that they trigger many more conversations on these important topics.

Editors: Shane Crotty and Rafi Ahmed

Additional Perspectives on Immune Memory and Vaccines: Great Debates available at www.cshperspectives.org

Copyright (C) 2018 Cold Spring Harbor Laboratory Press; all rights reserved; doi: 10.1101/cshperspect.a029371 Cite this article as Cold Spring Harb Perspect Biol 2018;10:a029371 
$\mathrm{H}^{\mathrm{u}}$ undreds of millions of people are infected annually by the four serotypes of dengue virus (DENV1-4), the etiological agents of dengue hemorrhagic fever/dengue shock syndrome (DHF/DSS) (Bhatt et al. 2013). Natural infection induces strongly neutralizing antibodies and lasting protective immunity to the homologous serotype. As highly effective live attenuated vaccines (LAVs) have been developed against other flaviviruses such as yellow fever and Japanese encephalitis viruses, the prospects for a dengue vaccine should be good (Murphy and Whitehead 2011). However, development of dengue vaccines is complicated by the presence of four distinct serotypes and the possibility of vaccine-enhanced severe dengue disease. Recently, several tetravalent dengue LAVs have been tested in human clinical trials with mixed results that highlight both the promise and problems of dengue vaccines (Rodriguez-Barraquer et al. 2014). Here, we review new information on the structural biology and immunology of DENVs and recent data from human natural infection and vaccination studies to identify best strategies for advancing dengue vaccines. Although antibodies and $\mathrm{T}$ cells both play important roles in DENV pathogenesis and protective immunity, our discussion here pertains to B-cell and antibody responses. Additionally, although alternative approaches to dengue vaccines exist (e.g., inactivated, subunit, vectored, DNA), we focus this discussion on LAVs.

\section{DENVs ARE HETEROGENEOUS AND DYNAMIC: IMPLICATIONS FOR VACCINE RESEARCH}

The four DENV serotypes each independently crossed into humans several hundred years ago from sylvatic ancestors (Vasilakis et al. 2011). After adapting to the human transmission cycle, each serotype further diverged into different genotypes with distinct geographical distributions (Holmes and Twiddy 2003). Many DENV strains commonly used for research and vaccine development are strains that are no longer in circulation and/or have been heavily passaged in laboratories. The interactions of some human antibodies with DENVs can be profoundly in- fluenced by genotype or passage history (Wahala et al. 2010; Sukupolvi-Petty et al. 2013). For example, the envelope (E) protein from DENV1 strain 16007 (isolated in Thailand in 1964) is currently included as a vaccine antigen. DENV1 16007 belongs to an extinct genotype and has mutations most likely derived from cell culture passage, which substantially alter neutralization sensitivity (Dowd et al. 2015). In a recent antigenic cartography study, this strain did not group with other DENV1 strains, further demonstrating its atypical properties (Katzelnick et al. 2015). Moving forward, we need to select the best representative contemporary strains for vaccine development and systematically assess whether vaccine-induced responses cover most major currently circulating strains.

The flavivirus virion contains two integral membrane proteins: $\mathrm{E}$ and premembrane $/ \mathrm{mem}$ brane $(\mathrm{prM} / \mathrm{M})$. During virus secretion from infected cells, prM is cleaved to generate mature infectious virions, which have a smooth surface of E protein dimers. In cell lines commonly used to propagate DENVs or dengue LAVs, prM processing is inefficient and variable, and virions released from cells are a heterogenous mixture of immature, partially mature, and fully mature virions containing variable amounts of unprocessed prM (Plevka et al. 2014). The maturation state of DENV alters the specific infectivity of the virus to different cell lines (Rodenhuis-Zybert et al. 2010) and the ability of antibodies to bind and neutralize the virus (Pierson and Diamond 2012). In partially immature virions, the highly conserved fusion peptide on $\mathrm{E}$ is exposed, resulting in increased binding and neutralization by abundant cross-reactive antibodies in immune sera. Currently, maturation state is not monitored and controlled in dengue vaccine research.

The surface of the flavivirus virion contains 90 antiparallel $\mathrm{E}$ dimers that are tightly packed to form a protein coat with icosahedral symmetry. Recent studies have established that, in some DENV strains, the protein envelope is flexible and antibodies are able to bind to cryptic epitopes and neutralize the virus (Lok et al. 2008). This phenomenon, termed virus breathing, is influenced by temperature, time of incubation, 
and DENV strain, and specific E protein mutations can have profound effects on virus breathing and exposure of antibody epitopes (Kuhn et al. 2015). No systematic studies have been conducted to assess how the extent of breathing varies according to serotype, genotype, strain, laboratory adaptation, and attenuation.

In summary, we now know that DENV serotypes are not monolithic. Rather, prM and $\mathrm{E}$ sequence variation, maturation state, and virus breathing have strong effects on epitope presentation and interactions with human antibodies. Most importantly, although we can account for serotype and genotype differences, we still do not know the maturation state and breathing/ flexibility of virions in people. Moving forward, basic and applied research on dengue vaccines has to be guided by existing information about the potential heterogeneity of DENV virions. We urgently need to define structural and biochemical properties of DENVs that circulate in humans.

\section{LESSONS LEARNED FROM NATURAL DENV INFECTION STUDIES}

Just as DENVs are not monolithic, the human immune response to DENV is variable, depending on serotype, strain, and the individual's prior flavivirus infection history. DENVs have antibody epitopes that are conserved or variable among serotypes. People exposed to their first DENV infection (primary infection) develop memory B cells (MBCs) and long-lived plasma cells (LLPCs) that produce antibodies that are cross-reactive or specific to the serotype of infection. Although cross-reactive antibodies are more abundant than type-specific antibodies in serum and MBCs, the potently neutralizing antibodies are mainly directed to the serotype of infection (de Alwis et al. 2012, 2014). After a primary infection, an individual is reliably protected against disease caused by the homologous serotype only.

We have also learned that a person experiencing a secondary DENV infection with a new serotype develops a neutralizing and protective antibody response that is fundamentally different from a primary infection-induced response
(Fig. 1). Secondary infections induce high levels of serotype cross-reactive antibodies that are, most likely, derived from MBCs from the first infection. These cross-reactive antibodies broadly neutralize two or more DENV serotypes, including serotypes not yet encountered (Corbett et al. 2015). Human cohort studies in dengue-endemic countries have also established that tertiary infections are nearly always mild or inapparent, implicating a protective role for broadly cross-neutralizing antibodies that develop after a second DENV infection (Olkowski et al. 2013).

The mere presence of anti-DENV antibodies is not sufficient for neutralization and protection. Importantly, not all neutralizing antibodies are protective to the same degree; thus, "quality" as well as "quantity" of anti-DENV neutralizing antibodies is paramount. As we discuss next, recent studies with human monoclonal antibodies (MAbs) derived from people exposed to primary or repeat infections have led to breakthroughs about the properties responsible for neutralization and protection.

Neutralizing and Protective Antibodies Induced after Primary DENV Infection

Serotype-specific human MAbs that strongly neutralize each serotype have been isolated from MBCs from people exposed to primary DENV infection (Beltramello et al. 2010; de Alwis et al. 2012). These antibodies bind to tertiary and quaternary structure epitopes on E present on intact virions or E homodimers (EDEs) but rarely on monomeric E protein (Fig. 2). These antibodies are protective in mouse models of DENV infection and disease, supporting a role for protective immunity in people (Teoh et al. 2012; Fibriansah et al. 2015). Investigators have used recombinant DENVs to determine whether epitopes recognized by these MAbs are also targets of the polyclonal-type-specific serum neutralizing antibody response. For example, the DENV2-specific human MAb 2D22 binds to a quaternary epitope that includes critical residues on $\mathrm{E}$ domain III (EDIII) of DENV2. The 2D22 epitope was successfully transplanted into DENV4 by creating a viable 
A.M. de Silva and E. Harris
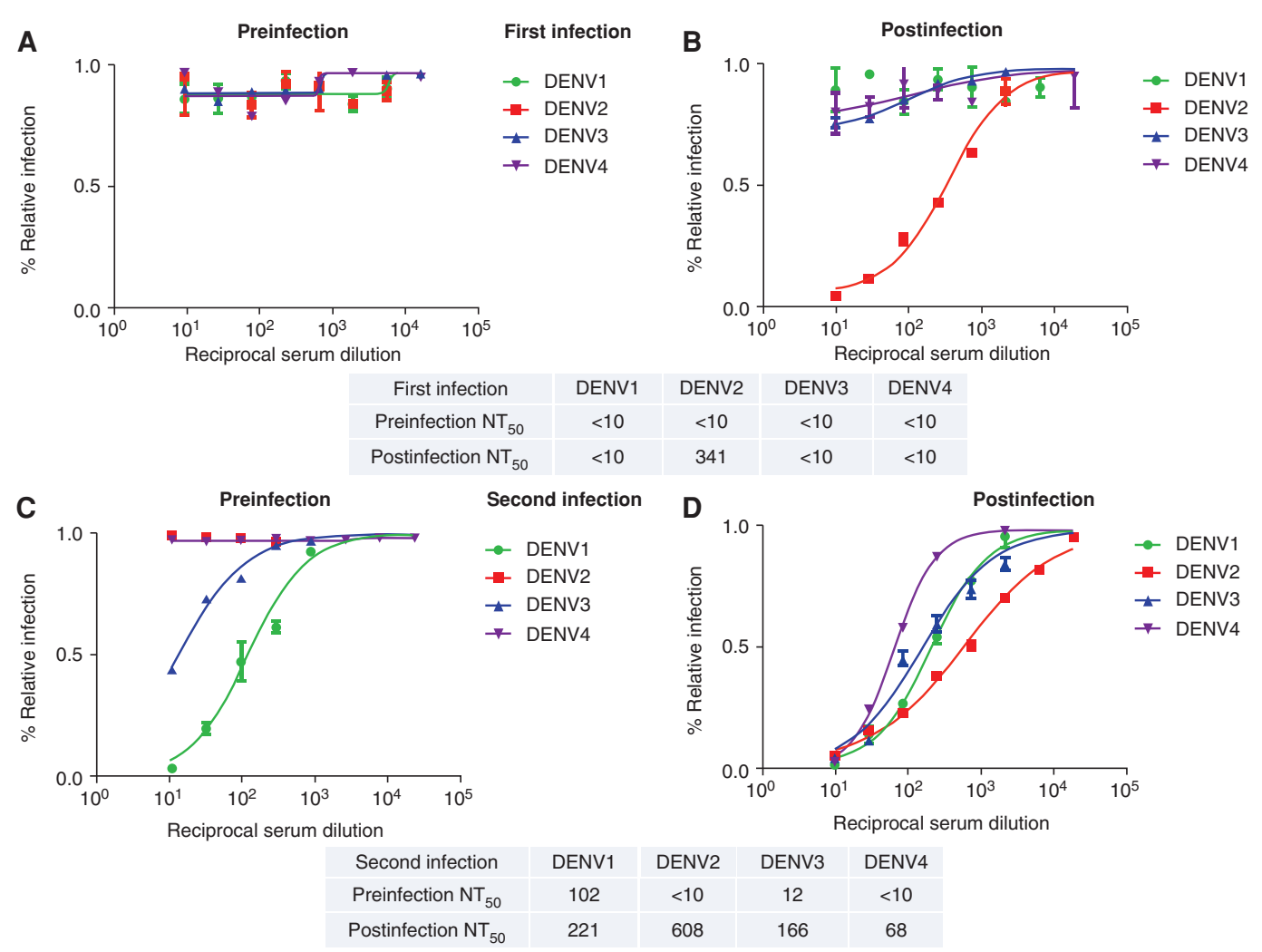

Figure 1. Neutralizing antibody response after a first versus a second natural dengue virus (DENV) infection. Shown are representative neutralization curves from a first $(A, B)$ versus a second $(C, D)$ DENV infection from children in a dengue cohort study in Nicaragua (Katzelnick et al. 2016). The preinfection $(A, C)$ and postinfection $(B, D)$ ( $>6$ months postinfection) neutralizing antibody responses are depicted, demonstrating a fundamentally different response in primary (i.e., first) versus second DENV infection. A table with the $50 \%$ neutralization titers $\left(\mathrm{NT}_{50}\right)$ is included below each set of graphs. Data were derived from neutralization assay performed in U937 human monocytic cells expressing the DENV attachment factor DC-SIGN and reporter viral particles representing DENV1, -2, -3, and -4 as described in Montoya et al. (2013).

recombinant virus containing EDI and EDII from DENV4 and EDIII from DENV2 (Gallichotte et al. 2015). Primary DENV2-immune sera efficiently neutralized the recombinant virus but not DENV4, demonstrating that the 2D22 epitope, and possibly other DENV2 epitopes in EDIII on the recombinant virus, are major targets of the human polyclonal neutralizing antibody response. A similar picture is emerging from studies with recombinant viruses displaying DENV1-, DENV3-, and DENV4type-specific quaternary epitopes (AM de Silva and RS Baric, unpubl.). We propose that quaternary epitopes that are unique to each serotype are the main targets of neutralizing and protective antibodies induced after primary DENV infection. Studies are ongoing to define how the primary neutralizing antibody response directed to these epitopes evolves over time and how the response varies depending on virus strain and individual infected.

Neutralizing and Protective Antibodies Induced after Secondary DENV Infection

Secondary DENV infections induce durable cross-neutralizing antibodies and cross-protective immunity. Several cross-neutralizing human 


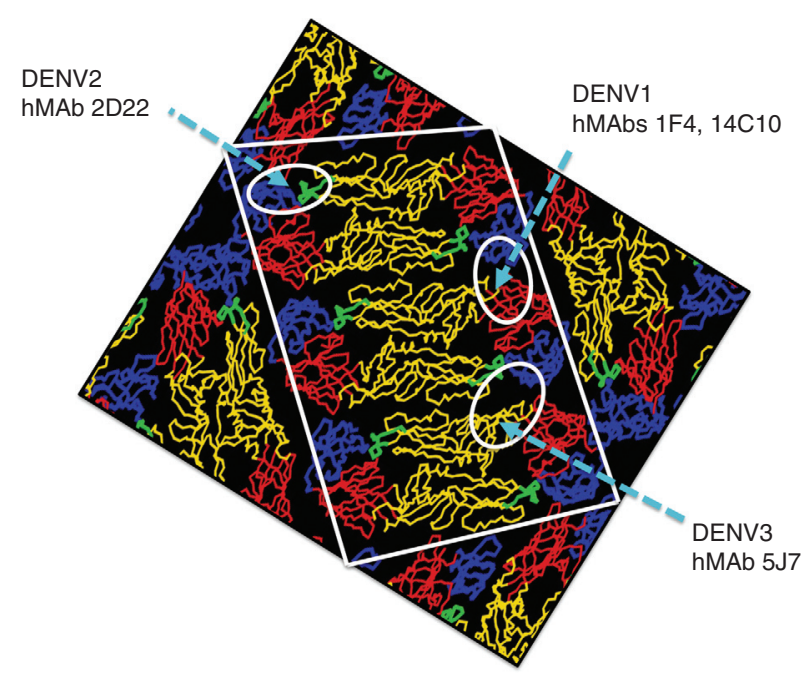

Figure 2. Location of quaternary epitopes recognized by serotype-specific human monoclonal antibodies (MAbs) that neutralize dengue viruses (DENVs). The DENV E protein has three domains, designated E domain I (EDI, red), E domain II (EDII, yellow), and E domain III (EDIII, blue). The viral fusion loop at the tip of EDII is indicated in green. E protein is present as a homodimer that lies flat on the surface of the mature virion. The white box depicts a raft of three E protein homodimers, which is the basic building block of the flavivirus envelope. Thirty rafts tightly pack to form a protein envelope with icosahedral symmetry. The approximate footprints of DENV1-specific (1F4 and 14C10), DENV2-specific (2D22), and DENV3-specific (5J7) human MAbs are circled. Note that the DENV1 MAb footprint encompasses EDI/II and EDIII from adjacent dimers. The DENV2 MAb footprint spans EDII and EDIII within a homodimer. The DENV3 MAb footprint includes contact residues from EDI, EDII, and EDIII of three different E protein subunits. (Adapted from www.purdue .edu/uns/images/kuhn.dengue1.jpeg.)

MAbs, which bind to an epitope near the bcloop on domain II of the E protein monomer, have been described (Smith et al. 2013; Tsai et al. 2013; Dejnirattisai et al. 2015). A novel class of serotype cross-reactive and strongly cross-neutralizing MAbs, which bind to quaternary epitopes on the E homodimer (EDE), was recently isolated from plasmablasts collected a few days after recovery from an acute secondary DENV infection (Dejnirattisai et al. 2015; Rouvinski et al. 2015). Other groups have also isolated plasmablasts from acute secondary dengue cases and mainly observed cross-reactive but weakly neutralizing antibodies (Fink 2012; Wrammert et al. 2012). The methods used to screen plasmablasts, the timing of sample collection, and individual variation and DENV infection histories may account for these differences. Furthermore, it is unclear whether the strongly cross-neutralizing MAbs isolated from acute-phase plasmablasts are maintained as MBCs and LLPCs. Several studies are currently in progress to define the origin and evolution of broadly neutralizing, cross-protective antibody responses. We propose that some low-affinity cross-reactive antibody-producing MBCs induced by primary infection are activated and affinity matured by repeat infections to generate higher-affinity cross-neutralizing antibodies such as the EDE antibodies. Development and evaluation of dengue vaccines must be grounded on the fundamentally different properties of neutralizing and protective antibodies that develop in naïve and partially immune individuals.

\section{LESSONS LEARNED FROM DENGUE LAVs}

LAVs have been successfully developed for several flaviviruses, and this approach holds much promise for a dengue vaccine. Because of the possibility of a monovalent vaccine inducing cross-reactive immunity that enhances replica- 
tion and disease caused by other DENV serotypes, all leading dengue vaccines are based on tetravalent formulations to induce simultaneous protective responses to all four serotypes. Three dengue LAVs, based on recombinant attenuated DENV serotypes (NIH) or recombinant yellow fever virus (Sanofi) or DENV2 (Takeda) constructs expressing the prM and $\mathrm{E}$ genes from the four serotypes (Murphy and Whitehead 2011), are currently at different stages of evaluation in phase 3 or 4 clinical trials. The leading candidate, Dengvaxia, developed by Sanofi Pasteur, has been approved for use in individuals above the age of 9 years in several countries (Guy and Jackson 2015). The first hurdle faced by developers was balanced replication of the four viruses in the tetravalent vaccine. Although all vaccines induced neutralizing antibodies to all four serotypes, some components have replicated better than others, and a strong positive correlation has been observed between vaccine virus replication and the magnitude and quality of neutralizing antibodies.

\section{Efficacy Data from Dengvaxia}

Currently, vaccine efficacy data is only available for Dengvaxia, which reduced the number of laboratory-confirmed cases of dengue disease in Asia and Latin America (Hadinegoro et al. 2015). However, vaccine efficacy varied by serotype, with higher efficacy rates against DENV3 and -4 than against DENV1 and -2 (Hadinegoro et al. 2015). The DENV3 and -4 components in the vaccine replicated better than the DENV1 and -2 components in preclinical animal studies and phase 1 human studies, establishing a correlation between vaccine virus replication and efficacy. Importantly, Dengvaxia efficacy was higher in participants with prior exposure to DENV than in participants dengue-naïve at baseline, demonstrating that it is easier to induce protective responses in DENV-primed individuals compared with naïve subjects (Hadinegoro et al. 2015). We have gained the following insights from this first dengue vaccine efficacy trial. First, just the presence of in vitro neutralizing antibodies is not sufficient for protection because many individuals experienced breakthrough infections despite having neutralizing antibodies to the breakthrough serotype. Second, the vaccine performed very differently in DENV-naïve versus -primed individuals, establishing the profound impact of immunological memory on vaccine performance.

\section{A CONTEMPORARY FRAMEWORK FOR DENGUE VACCINE DEVELOPMENT}

Moving forward, we strongly recommend separating the evaluation of vaccine performance in DENV-naive and -primed individuals, as the barriers to inducing protective immunity and the specific properties of neutralizing/protective antibodies are fundamentally different in these two populations (Fig. 3). All dengue vaccine trials must be designed to obtain separate safety and efficacy data for these two populations because it is not possible to extrapolate results from one group to the other (Rodriguez-Barraquer et al. 2015;Russell and Halstead 2016).

\section{Effective Vaccination of DENV-Naïve Individuals}

The population with the greatest need for a dengue vaccine is young children living in endemic countries. The majority of children will be DENV-naive at vaccination. What are the likely properties of vaccine-induced protective antibodies in this population? In people exposed to primary DENV infections, the neutralizing and protective antibody response is dominated by type-specific antibodies; thus, cross-reactive neutralizing antibodies induced by unbalanced and robust replication of one or two serotypes in a tetravalent vaccine are unlikely to provide durable protection against all four serotypes in this naïve population. In fact, DENV-naïve subjects who received Dengvaxia developed high levels of type-specific neutralizing antibodies to DENV4, whereas the DENV1, -2 , and -3 responses were dominated by crossreactive neutralizing antibodies (Henein et al. 2017), possibly derived from the DENV4 component that replicated the best in vaccinees. We 

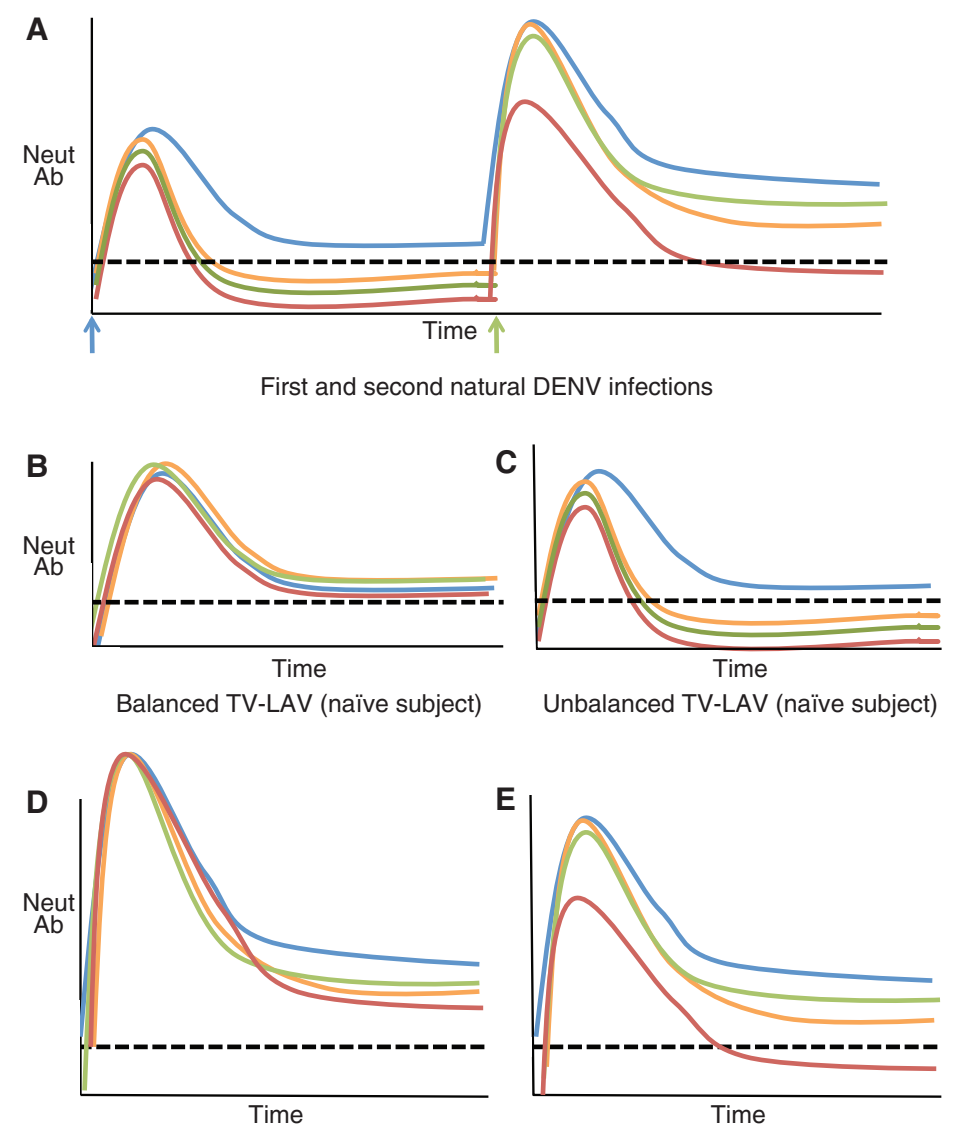

Balanced TV-LAV (preimmune subject) Unbalanced TV-LAV (preimmune subject)

Figure 3. Effect of preexisting dengue virus (DENV) immunity on antibody responses to balanced and unbalanced tetravalent live attenuated (TV-LAV) vaccines. (A) Levels of neutralizing antibodies after first and second natural DENV infections with different serotypes. After the first infection, there is a rapid increase in neutralizing antibodies to all four serotypes, which decline over time. Type-specific antibodies to the homologous serotype (blue) are maintained at higher levels, whereas antibodies to heterologous serotypes decline and are maintained at lower levels or become undetectable. A second infection with a new serotype (green) leads to high levels of neutralizing antibodies to the four serotypes, which decline over time. Compared with the first infection, higher levels of DENV serotype cross-reactive neutralizing are maintained after a second infection. Neutralizing antibody responses in DENV-naïve subjects who received a balanced $(B)$ or unbalanced $(C)$ TVLAV. A vaccine with balanced replication of all four serotypes $(B)$ will induce durable, type-specific neutralizing antibody responses to the four serotypes. After vaccination with an unbalanced TV-LAV $(C)$, dominant replication of one serotype will, initially, induce neutralizing antibodies to all four serotypes, which decline over time. Type-specific antibodies to the dominant serotype will be maintained, whereas antibodies to other serotypes will decline to low or undetectable levels. Neutralizing antibody responses in DENV preimmune subjects who received a balanced $(D)$ or unbalanced $(E)$ TV-LAV. A vaccine with balanced replication of all four serotypes $(D)$ will induce durable, type-specific, and cross-reactive neutralizing antibody responses to the four serotypes. A vaccine with unbalanced, dominant replication of one serotype $(E)$ will be similar to a secondary natural secondary DENV infection. The unbalanced vaccine will induce variable levels of type-specific and crossreactive neutralizing antibodies to each of the four serotypes. The four colored lines represent evolving antibody responses to each of the four serotypes of DENV. The dashed black line represents a hypothetical neutralizing titer required for protection. In reality, the absolute titer for protection is likely to be different for each serotype and also influenced by the quality of neutralizing antibodies. Neut Ab, Neutralizing antibody. 
A.M. de Silva and E. Harris

propose that balanced replication leading to type-specific antibodies that target quaternary epitopes in each serotype is the key to effective vaccination of naïve individuals.

\section{Effective Vaccination of DENV-Primed Individuals}

Secondary DENV infections result in activation of MBCs and development and expansion of cross-reactive antibodies that broadly neutralize multiple DENV serotypes, driven by the sequential infection and robust replication of two different serotypes of DENV. We propose that a similar mechanism is responsible for the superior performance of tetravalent LAVs in DENV-primed individuals. In a subject with preexisting DENV-specific MBCs, even unbalanced replication of one or two vaccine components is likely to activate MBCs and expand somatically mutated higher-affinity cross-reactive clones with capacity to broadly neutralize multiple serotypes.

\section{HOW SAFE ARE DENGUE VACCINES?}

A major concern regarding dengue vaccines is the potential for increased risk of enhanced disease in vaccine recipients who mount an insufficient/suboptimal immune response or after initially protective levels of antibodies wane to potentially enhancing levels. These concerns are based on epidemiological studies that have shown that a second infection with a new serotype or natural infection of 6- to 12-month-old infants of DENV-immune mothers leads to increased risk of severe disease (Burke et al. 1988; Kliks et al. 1988). This phenomenon is posited to be a result, in part, of antibody-dependent enhancement (ADE), whereby nonneutralizing antibodies to a previous DENV infection bind to the new serotype and facilitate its entry into target Fc $\gamma$ receptor (Fc $\gamma \mathrm{R}$ )-bearing cells, leading to higher viremia and immune activation (Halstead 1988). However, studies of ADE in human populations remain controversial. Some studies have shown that sera from children who develop more severe disease are more enhancing in vitro (Kliks et al. 1988; Chau et al.
2008); other studies have not found this to be the case (Laoprasopwattana et al. 2005; Libraty et al. 2009). Certainly, serotype cross-reactive antibodies (and even serotype-specific antibodies at high enough dilution) can cause ADE in vitro in numerous Fc $\gamma$ R-bearing cells; the issue remains whether in vitro results can be extrapolated to human studies and, currently, we do not have robust serological assays for accurately predicting risk of $\mathrm{ADE}$ and severe disease in people. The most convincing evidence for ADE has come from animal studies. Prior administration of subneutralizing levels of polyclonal serum or MAbs can enhance DENV replication in nonhuman primates (Goncalvez et al. 2007) and cause a lethal vascular leak syndrome in mouse models (Balsitis et al. 2010; Zellweger et al. 2010). Further studies with mice passively administered human DENV immune sera showed that DENV cross-reactive antibodies that bound to recombinant $\mathrm{E}$ and prM proteins were responsible for $\mathrm{ADE}$ in the lethal mouse model (de Alwis et al. 2014). However, it is still unclear exactly how such animal studies translate into severe disease in humans, although a new study demonstrates that a specific range of low-titer antibodies predicts risk of severe dengue disease in a pediatric cohort study in Nicaragua (L Katzelnick and E Harris, unpubl.).

The most direct evidence about vaccine safety emerges from the clinical trials themselves. During the long-term follow-up period of the Sanofi phase 3 trial in Asia, children who received the vaccine had an increased risk of hospitalization because of dengue compared with the placebo group (Hadinegoro et al. 2015). As children were more likely to be DENV-naïve compared with adults at the time of vaccination, the most parsimonious explanation for this safety signal is that vaccination of naïve individuals predisposed them to more severe disease. The increased risk of hospitalization peaked in the second year after vaccination, demonstrating that immunity to flaviviruses and potential susceptibility to ADE is a "moving target" as the immune response wanes. It is also possible that other factors such as age and "temporal clustering" of cases are responsible for the 
increased number of hospitalized cases (Guy and Jackson 2015). The entire dengue vaccine community will learn a great deal from the intense studies currently in progress to better understand the overall efficacy patterns and safety profile of Dengvaxia (Guy and Jackson 2015).

\section{FUTURE DIRECTIONS}

Immune correlates of protection and vaccine efficacy are urgently needed. Several recent studies confirm that neutralizing antibody titer is associated with protection from symptomatic disease (Endy et al. 2004; Katzelnick et al. 2016); improved correlates will emerge as epitope-specific diagnostics become available. However, it seems likely that particular immune correlates will be specific for each DENV serotype and vaccine; it is also possible that the threshold of neutralizing antibodies or other immune correlates that protect against subsequent infection is also influenced by the force of infection of a given epidemic (Katzelnick et al. 2016). Furthermore, it must be kept in mind that neutralization titers vary substantially across laboratories and between methods, with numerous assay parameters significantly affecting the outcome (Thomas et al. 2009), adding yet another layer of complexity.

Another discussion has been whether a vaccine containing fewer than four serotypes could be safe and efficacious. This would depend on generating effective protective cross-reactive responses, and, until we know how to elicit such responses in all vaccinees, it is critical to include LAV or immunogens from all four serotypes. Another approach under development is bivalent vaccine candidates, wherein key neutralizing epitopes from one serotype are transplanted to another serotype while maintaining protective epitopes in the recipient virus (Gallichotte et al. 2015; Messer et al. 2016). In this case, the determining factor is the universality of the particular epitope(s) in question. Finally, a strategy under active investigation by several groups is the construction of scaffolded vaccines, whereby only the epitope(s) of interest is displayed in the proper configuration on a neutral scaf- fold or, alternatively, development of methods to mask epitopes that may generate "unwanted" nonneutralizing/nonprotective antibodies. These latter approaches have been shown with other targets, such as HIV or respiratory syncytial virus (RSV) (McLellan et al. 2011); the challenge is to apply these methods to solve the dengue vaccine conundrum.

\section{CONCLUSIONS}

The development of the dengue LAVs currently in clinical trials started several decades ago. In the interim, we have learned a lot about the structural properties of DENVs and the human antibody response to natural infections. We will gain unprecedented insight into how dengue LAVs can be safe and effective from the current clinical trials. Although we acknowledge the difficulty of the long timeline from initial formulation of vaccine to clinical trials, in this review we have provided a framework for incorporating new information about DENVs and the human antibody response to these viruses to advance the development of dengue vaccines.

\section{ACKNOWLEDGMENTS}

The authors thank Leah Katzelnick for thoughtful comments on the manuscript, members of the de Silva and Baric laboratories for stimulating discussions and insights, and Magelda Montoya for her contribution to Figure 1. This work was supported in part by grants from the National Institutes of Health R01 AI125198-01 (A.M.d.S.), R01 AI107731 (A.M.d.S.), and P01 AI106695 (E.H.).

\section{REFERENCES}

Balsitis SJ, Williams KL, Lachica R, Flores D, Kyle JL, Mehlhop E, Johnson S, Diamond MS, Beatty PR, Harris E. 2010. Lethal antibody enhancement of dengue disease in mice is prevented by Fc modification. PLoS Pathog 6: e1000790.

Beltramello M, Williams KL, Simmons CP, MacAgno A, Simonelli L, Quyen NTH, Sukupolvi-Petty S, NavarroSanchez E, Young PR, de Silva AM, et al. 2010. The human immune response to dengue virus is dominated by highly cross-reactive antibodies endowed with neutralizing and enhancing activity. Cell Host Microbe 8: 271-283. 
A.M. de Silva and E. Harris

Bhatt S, Gething PW, Brady OJ, Messina JP, Farlow AW, Moyes CL, Drake JM, Brownstein JS, Hoen AG, Sankoh O, et al. 2013. The global distribution and burden of dengue. Nature 496: 504-507.

Burke DS, Nisalak A, Johnson DE, Scott RM. 1988. A prospective study of dengue infections in Bangkok. Am J Trop Med Hyg 38: 172-180.

Chau TNB, Quyen NTH, Thuy TT, Tuan NM, Hoang DM, Dung NTP, Lien LB, Quy NT, Hieu NT, Hieu LTM, et al. 2008. Dengue in Vietnamese infants-Results of infection-enhancement assays correlate with age-related disease epidemiology, and cellular immune responses correlate with disease severity. J Infect Dis B 98: 516-524.

Corbett KS, Katzelnick L, Tissera H, Amerasinghe A, de Silva AD, de Silva AM. 2015. Preexisting neutralizing antibody responses distinguish clinically inapparent and apparent dengue virus infections in a Sri Lankan pediatric cohort. J Infect Dis 211: 590-599.

de Alwis R, Smith SA, Olivarez NP, Messer WB, Huynh JP, Wahala WMPB, White LJ, Diamond MS, Baric RS, Crowe JE, et al. 2012. Identification of human neutralizing antibodies that bind to complex epitopes on dengue virions. Proc Natl Acad Sci 109: 7439-7444.

de Alwis R, Williams KL, Schmid MA, Lai CY, Patel B, Smith SA, Crowe JE, Wang WK, Harris E, de Silva AM. 2014. Dengue viruses are enhanced by distinct populations of serotype cross-reactive antibodies in human immune sera. PLoS Pathog 10: e1004386.

Dejnirattisai W, Wongwiwat W, Supasa S, Zhang X, Dai X, Rouvinsky A, Jumnainsong A, Edwards C, Quyen NTH, Duangchinda T, et al. 2015. A new class of highly potent, broadly neutralizing antibodies isolated from viremic patients infected with dengue virus. Nat Immunol 16: 785.

Dowd KA, DeMaso CR, Pierson TC. 2015. Genotypic differences in dengue virus neutralization are explained by a single amino acid mutation that modulates virus breathing. mBio 6: $\mathrm{e} 01559$.

Endy TP, Nisalak A, Chunsuttitwat S, Vaughn DW, Green S, Ennis FA, Rothman AL, Libraty DH. 2004. Relationship of preexisting dengue virus (DV) neutralizing antibody levels to viremia and severity of disease in a prospective cohort study of DV infection in Thailand. Infect Dis 189: 990-1000.

Fibriansah G, Ibarra KD, Ng TS, Smith SA, Tan JL, Lim Xn, Ooi JSG, Kostyuchenko VA, Wang J, de Silva AM, et al. 2015. Cryo-EM structure of an antibody that neutralizes dengue virus type 2 by locking E protein dimers. Science 349: 88-91.

Fink K. 2012. Origin and function of circulating plasmablasts during acute viral infections. Front Immunol 3: 78.

Gallichotte EN, Widman DG, Yount BL, Wahala WM, Durbin A, Whitehead S, Sariol CA, Crowe JE. 2015. A new quaternary structure epitope on dengue virus serotype 2 is the target of durable type-specific neutralizing antibodies. mBio 6: 1-8.

Goncalvez AP, Engle RE, St Claire M, Purcell RH, Lai CJ. 2007. Monoclonal antibody-mediated enhancement of dengue virus infection in vitro and in vivo and strategies for prevention. Proc Natl Acad Sci 104: 9422-9427.

Guy B, Jackson N. 2015. Dengue vaccine: Hypotheses to understand CYD-TDV-induced protection. Nat Rev Microbiol 14: 45-54.
Hadinegoro SR, Arredondo-García JL, Capeding MR, Deseda C, Chotpitayasunondh T, Dietze R, Ismail HIHM, Reynales H, Limkittikul K, Rivera-Medina DM, et al. 2015. Efficacy and long-term safety of a dengue vaccine in regions of endemic disease. New Engl J Med 373: 1195 1206.

Halstead SB. 1988. Pathogenesis of dengue: Challenges to molecular biology. Science 239: 476-481.

Henein S, Swanstrom J, Byers AM, Moser JM, Shaik SF, Bonaparte M, Jackson N, Guy B, Baric R, de Silva AM. 2017. Dissecting antibodies induced by a chimeric yellow fever-dengue, live-attenuated, tetravalent dengue vaccine (CYD-TDV) in naïve and dengue-exposed individuals. $J$ Infect Dis 215: 351-358.

Holmes EC, Twiddy SS. 2003. The origin, emergence and evolutionary genetics of dengue virus. Infect Genet Evol 3: $19-28$.

Katzelnick LC, Fonville JM, Gromowski GD, Bustos Arriaga J, Green A, James SL, Lau L, Montoya M, Wang C, VanBlargan LA, et al. 2015. Dengue viruses cluster antigenically but not as discrete serotypes. Science 349: $1338-$ 1343.

Katzelnick LC, Montoya M, Gresh L, Balmaseda A, Harris E. 2016. Neutralizing antibody titers against dengue virus correlate with protection from symptomatic infection in a longitudinal cohort. Proc Natl Acad Sci 113: 728-733.

Kliks SC, Nimmanitya S, Nisalak A, Burke DS. 1988. Evidence that maternal dengue antibodies are important in the development of dengue hemorrhagic fever in infants. Am J Trop Med Hyg 38: 411-419.

Kuhn RJ, Dowd KA, Beth Post C, Pierson TC. 2015. Shake, rattle, and roll: Impact of the dynamics of flavivirus particles on their interactions with the host. Virology 479480: 508-517.

Laoprasopwattana K, Libraty DH, Endy TP, Nisalak A, Chunsuttiwat S, Vaughn DW, Reed G, Ennis FA, Rothman AL, Green S. 2005. Dengue virus (DV) enhancing antibody activity in preillness plasma does not predict subsequent disease severity or viremia in secondary DV infection. J Infect Dis 192: 510-519.

Libraty DH, Acosta LP, Tallo V, Segubre-Mercado E, Bautista A, Potts JA, Jarman RG, Yoon IK, Gibbons RV, Brion JD, et al. 2009. A prospective nested case-control study of dengue in infants: Rethinking and refining the antibody-dependent enhancement dengue hemorrhagic fever model. PLoS Med 6: e1000171.

Lok SM, Kostyuchenko V, Nybakken GE, Holdaway HA, Battisti AJ, Sukupolvi-Petty S, Sedlak D, Fremont DH, Chipman PR, Roehrig JT, et al. 2008. Binding of a neutralizing antibody to dengue virus alters the arrangement of surface glycoproteins. Nat Struct Mol Biol 15:312-317.

McLellan JS, Correia BE, Chen M, Yang Y, Graham BS, Schief WR, Kwong PD. 2011. Design and characterization of epitope-scaffold immunogens that present the motavizumab epitope from respiratory syncytial virus. J Mol Biol 409: 853-866.

Messer WB, Yount BL, Royal SR, de Alwis RA, Widman DG, Smith SA, Crowe JE Jr, Pfaff JM, Kahle KM, Doranz BJ, et al. 2016. Functional transplant of a DENV3-specific human monoclonal antibody epitope into DENV1. J Virol 90: 5090-5097. 
Montoya M, Gresh L, Mercado JC, Williams KL, Vargas MJ, Gutierrez G, Kuan G, Gordon A, Balmaseda A, Harris E. 2013. Symptomatic versus inapparent outcome in repeat dengue virus infections is influenced by the time interval between infections and study year. PLoS Negl Trop Dis 7: e2357.

Murphy BR, Whitehead SS. 2011. Immune response to dengue virus and prospects for a vaccine. Ann Rev Immunol 29: 587-619.

Olkowski S, Forshey BM, Morrison AC, Rocha C, Vilcarromero S, Halsey ES, Kochel TJ, Scott TW, Stoddard ST. 2013. Reduced risk of disease during postsecondary dengue virus infections. J Infect Dis 208: 1026-1033.

Pierson TC, Diamond MS. 2012. Degrees of maturity: The complex structure and biology of flaviviruses. Curr Opin Virol 2: $168-175$.

Plevka P, Battisti AJ, Sheng J, Rossmann MG. 2014. Mechanism for maturation-related reorganization of flavivirus glycoproteins. J Struct Biol 185: 27-31.

Rodenhuis-Zybert IA, Wilschut J, Smit JM. 2010. Dengue virus life cycle: Viral and host factors modulating infectivity. Cell Molec Life Sci 67: 2773-2786.

Rodriguez-Barraquer I, Mier-y-Teran-Romero L, Schwartz IB, Burke DS, Cummings DAT. 2014. Potential opportunities and perils of imperfect dengue vaccines. Vaccine 32: $514-520$.

Rodriguez-Barraquer I, Mier-y-Teran-Romero L, Ferguson N, Burke DS, Cummings DA. 2015. Differential efficacy of dengue vaccine by immune status. Lancet 385: 1726.

Rouvinski A, Guardado-Calvo P, Barba-Spaeth G, Duquerroy S, Vaney MC, Kikuti CM, Navarro Sanchez ME, Dejnirattisai W, Wongwiwat W, Haouz A, et al. 2015. Recognition determinants of broadly neutralizing human antibodies against dengue viruses. Nature 520: 109-113.

Russell PK, Halstead SB. 2016. Challenges to the design of clinical trials for live-attenuated tetravalent dengue vaccines. PLoS Negl Trop Dis 10: e0004854.

Smith SA, de Alwis AR, Kose N, Harris E, Ibarra KD, Kahle KM, Pfaff JM, Xiang X, Doranz BJ, de Silva AM, et al. 2013. The potent and broadly neutralizing human dengue virus-specific monoclonal antibody 1 C19 reveals a unique cross-reactive epitope on the bc loop of domain II of the envelope protein. mBio 4: e00873.

Sukupolvi-Petty S, Brien JD, Austin SK, Shrestha B, Swayne S, Kahle K, Doranz BJ, Johnson S, Pierson TC, Fremont $\mathrm{DH}$, et al. 2013. Functional analysis of antibodies against dengue virus type 4 reveals strain-dependent epitope exposure that impacts neutralization and protection. J Virol 87: 8826-8842.

Teoh EP, Kukkaro P, Teo EW, Lim APC, Tan TT, Yip A, Schul W, Aung M, Kostyuchenko VA, Leo YS, et al. 2012. The structural basis for serotype-specific neutralization of dengue virus by a human antibody. Sci Transl Med 4: 139 ra83.

Thomas SJ, Nisalak A, Anderson KB, Libraty DH, Kalayanarooj S, Vaughn DW, Putnak R, Gibbons RV, Jarman R, Endy TP. 2009. Dengue plaque reduction neutralization test (PRNT) in primary and secondary dengue virus infections: How alterations in assay conditions impact performance. Am J Trop Med Hyg 81: 825-833.

Tsai WY, Lai CY, Wu YC, Lin HE, Edwards C, Jumnainsong A, Kliks S, Halstead S, Mongkolsapaya J, Screaton GR, et al. 2013. High-avidity and potently neutralizing crossreactive human monoclonal antibodies derived from secondary dengue virus infection. J Virol 87: 12562-12575.

Vasilakis N, Cardosa J, Hanley KA, Holmes EC, Weaver SC. 2011. Fever from the forest: Prospects for the continued emergence of sylvatic dengue virus and its impact on public health. Nat Rev Microbiol 9: 532-541.

Wahala WMPB, Donaldson EF, de Alwis R, Accavitti-Loper MA, Baric RS, de Silva AM. 2010. Natural strain variation and antibody neutralization of dengue serotype 3 viruses. PLoS Pathog 6: e1000821.

Wrammert J, Onlamoon N, Akondy RS, Perng GC, Polsrila K, Chandele A, Kwissa M, Pulendran B, Wilson PC, Wittawatmongkol O, et al. 2012. Rapid and massive virusspecific plasmablast responses during acute dengue virus infection in humans. J Virol 86: 2911-2918.

Zellweger RM, Prestwood TR, Shresta S. 2010. Enhanced infection of liver sinusoidal endothelial cells in a mouse model of antibody-induced severe dengue disease. Cell Host Microbe 7: 128-139. 


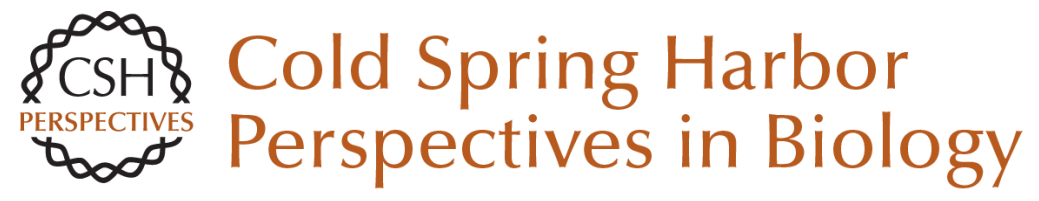

\section{Which Dengue Vaccine Approach Is the Most Promising, and Should We Be Concerned about Enhanced Disease after Vaccination?: The Path to a Dengue Vaccine: Learning from Human Natural Dengue Infection Studies and Vaccine Trials}

Aravinda M. de Silva and Eva Harris

Cold Spring Harb Perspect Biol 2018; doi: 10.1101/cshperspect.a029371 originally published online July 17,2017

\section{Subject Collection Immune Memory and Vaccines: Great Debates}

Is There Natural Killer Cell Memory and Can It Be Harnessed by Vaccination?: Can Natural Killer and CD8 T Cells Switch Jobs?

Christine A. Biron and Marcus Altfeld

Is There Natural Killer Cell Memory and Can It Be Harnessed by Vaccination?: Vaccination

Strategies Based on NK Cell and ILC Memory

Megan A. Cooper, Todd A. Fehniger and Marco

Colonna

Is It Possible to Develop Cancer Vaccines to Neoantigens, What Are the Major Challenges, and How Can These Be Overcome?: Neoantigens as Vaccine Targets for Cancer

Haydn T. Kissick

Is It Possible to Develop Cancer Vaccines to Neoantigens, What Are the Major Challenges, and How Can These Be Overcome?: Neoantigens:

Nothing New in Spite of the Name

Olivera J. Finn and Hans-Georg Rammensee
Is There Natural Killer Cell Memory and Can It Be Harnessed by Vaccination?: NK Cell Memory and Immunization Strategies against Infectious

Diseases and Cancer Joseph C. Sun and Lewis L. Lanier

Is There Natural Killer Cell Memory and Can It Be Harnessed by Vaccination?: Natural Killer Cells in Vaccination

Harold R. Neely, Irina B. Mazo, Carmen Gerlach, et al.

Is It Possible to Develop Cancer Vaccines to Neoantigens, What Are the Major Challenges, and How Can These Be Overcome?: Targeting the Right Antigens in the Right Patients Stephen P. Schoenberger

Which Dengue Vaccine Approach Is the Most Promising, and Should We Be Concerned about Enhanced Disease after Vaccination?: There Is Only One True Winner Scott B. Halstead

For additional articles in this collection, see http://cshperspectives.cshlp.org/cgi/collection/

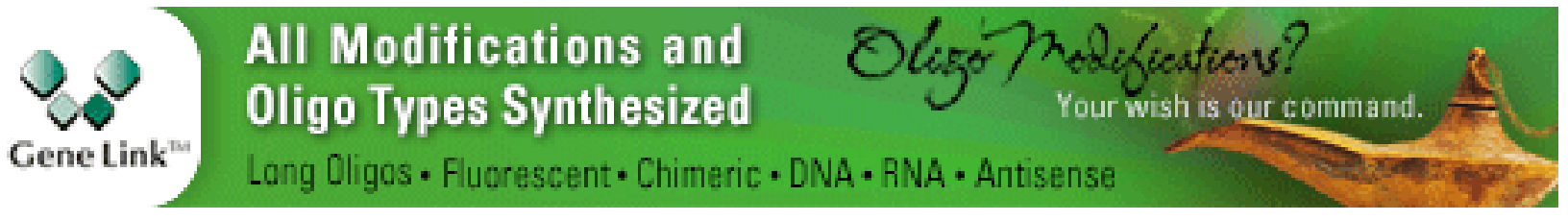


Which Dengue Vaccine Approach Is the Most Promising, and Should We Be Concerned about Enhanced Disease after Vaccination?: The Challenges of a Dengue Vaccine

Gavin Screaton and Juthathip Mongkolsapaya

Which Dengue Vaccine Approach Is the Most Promising, and Should We Be Concerned about Enhanced Disease after Vaccination?: The Path to a Dengue Vaccine: Learning from Human Natural Dengue Infection Studies and Vaccine Trials Aravinda M. de Silva and Eva Harris

Is It Possible to Develop a "Universal" Influenza Virus Vaccine?: Potential for a Universal Influenza Vaccine James E. Crowe, Jr.

Is It Possible to Develop a "Universal" Influenza Virus Vaccine?: Outflanking Antibody Immunodominance on the Road to Universal Influenza Vaccination

Davide Angeletti and Jonathan W. Yewdell
Which Dengue Vaccine Approach Is the Most Promising, and Should We Be Concerned about Enhanced Disease after Vaccination?: Questions Raised by the Development and Implementation of Dengue Vaccines: Example of the Sanofi Pasteur Tetravalent Dengue Vaccine Bruno Guy

Which Dengue Vaccine Approach Is the Most Promising, and Should We Be Concerned about Enhanced Disease after Vaccination?: The Risks of Incomplete Immunity to Dengue Virus Revealed by Vaccination

Stephen S. Whitehead and Kanta Subbarao

Is It Possible to Develop a "Universal" Influenza Virus Vaccine?: Immunogenetic Considerations Underlying B-Cell Biology in the Development of a Pan-Subtype Influenza A Vaccine Targeting the Hemagglutinin Stem Sarah F. Andrews, Barney S. Graham, John R. Mascola, et al.

Is It Possible to Develop a "Universal" Influenza Virus Vaccine?: Potential Target Antigens and Critical Aspects for a Universal Influenza Vaccine Florian Krammer, Adolfo García-Sastre and Peter Palese

For additional articles in this collection, see http://cshperspectives.cshlp.org/cgi/collection/

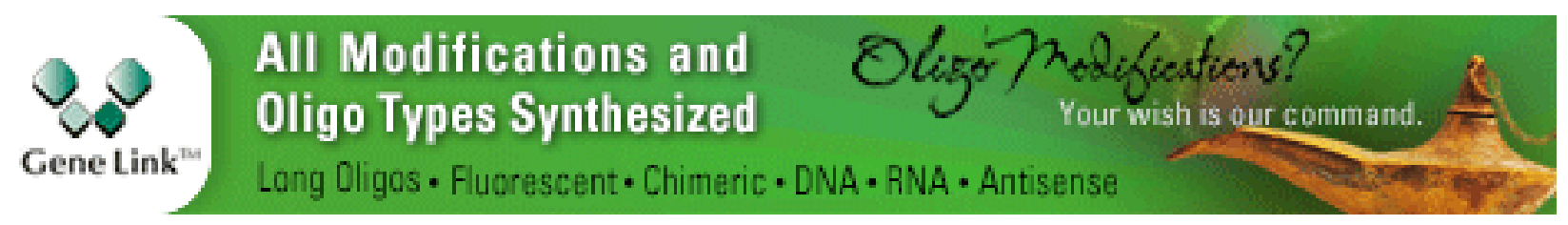

